FACTA UNIVERSITATIS

Series: Mechanical Engineering Vol. 17, No 1, 2019, pp. 75 - 85

https://doi.org/10.22190/FUME190115006W

Original scientific paper

\title{
ENERGY LOSS AND WEAR IN SPHERICAL OBLIQUE ELASTIC IMPACTS
}

\section{Emanuel Willert}

\author{
Technische Universität Berlin, Berlin, Germany
}

\begin{abstract}
Percussive and erosive wear by repetitive impacting of solid particles damages surfaces even at low impact velocities. As the impact wear is often directly related to the energy loss during the collision and therefore to the coefficients of normal and tangential restitution, in the present study the oblique low-velocity impact of a rigid sphere onto an elastic half-space is analyzed based on the known respective contact-impact solution and with regard to the energy loss during the impact. Simple analytic expressions are derived for the total impact wear volume. It is found that the portion of kinetic energy lost in frictional dissipation has a well-located maximum for configurations with weak forward pre-spin. The distribution of frictional dissipation over the contact area has a complex dependence on the impact parameters. For pronounced local slip (e.g. due to a small coefficient of friction) the dissipation accumulated over the collision is localized in the center of impact whereas for dominance of sticking, most energy is lost away from the center.
\end{abstract}

Key Words: Elastic Impacts, Friction, Energy Dissipation, Wear

\section{INTRODUCTION}

Impact wear, i.e. material degradation due to the repetitive impacting of solid particles onto a surface is a serious source of damage and failure in several technical systems like steam generator tubes [1], mining machinery [2] and others. Several studies, starting more than fifty years ago, have been dealing with the erosion of a surface by a stream of solid particles [3-6]. However, due to the complexity of the occurring wear mechanisms and the mathematical difficulty of the contact mechanical description for the impact itself, the problem remains far from being fully understood.

Received January 15, 2019 / Accepted March 07, 2019

Corresponding author: Emanuel Willert

Technische Universität Berlin, Sekr. C8-4, Straße des 17. Juni 135, 10623 Berlin, Germany

E-mail: e.willert@tu-berlin.de 
The first rigorously analyzed contact-impact problem is the low-velocity normal impact of perfectly elastic, perfectly smooth spheres, solved by Hertz [7]. The Hertzian normal impact solution has later been generalized to incorporate adhesion [8], plasticity [9], wave propagation [10] and surface roughness [11]. The frictional oblique impact problem of elastic spheres was solved by Maw, Barber \& Fawcett (MBF, [12]), based on the contact solution for the frictional tangential contact of elastic spheres under varying oblique loading histories by Mindlin and Deresiewicz [13]. The outcomes of the MBF theory were demonstrated to be in very good agreement with experimental results if the impact behavior is close to being perfectly elastic [14, 15]. An equivalent but computationally simpler formulation of the MBF solution within the framework of the method of dimensionality reduction [16] was published recently [17]. Moreover, there are several rigid-body models of oblique impacts [18, 19]. However, as there are only two regimes of contact in rigid-body dynamics - stick and gross sliding - these models are necessarily wrong in the partial slip regime.

A common approach to characterizing the impact wear behavior is to study the loss of kinetic energy during the impact [1]. Thereby good agreement has been reported between energy-based models and erosive wear results from the literature [20, 1]. This is in correlation with the classical wear law by Archard and Hirst [21] for adhesive and Khrushchov and Babichev [22] for abrasive wear, according to which the wear intensity is proportional to the normal load and the relative tangential velocity of the contacting surfaces. If we additionally apply the Amontons-Coulomb law of friction, the wear intensity is proportional to the power of frictional energy dissipation, as reported by Honda and Yamada [23]. The energy-based approach to studying erosive wear was also used by Argatov et al. [24], who, nevertheless, only considered sliding contact during the impact and neglected the contribution of spin.

Thus, in the present study, the loss of kinetic energy due to frictional dissipation will be analyzed for the oblique impact of elastic spheres, based on the MBF model for the contact-impact problem. First, in a general analysis, the rebound velocities and the loss of kinetic energy are calculated in terms of the tangential coefficient of restitution. After that, this restitution coefficient is determined within the framework of the MBF approach. After a visualization of the main results for the total loss of kinetic energy, the distribution of frictional dissipation over the contact area is analyzed. A short discussion closes the manuscript.

\section{GENERAL ANALYSIS}

Let us look into the problem depicted in Fig. 1: A rigid sphere of mass $m$, radius $R$ and moment of inertia $J$ impacts onto an elastic half-space with initial velocities $v_{x 0}$ and $v_{z 0}$ and initial angular velocity $\omega_{0}$. Without loss of generality we can assume that $v_{x 0}>0$; for brevity let us introduce spin $s=R \omega$. The impact shall be short and the macroscopic dynamics shall be determined by point contact forces in normal and tangential direction at the point of first contact $K$.

Note that the more general case of colliding elastic spheres in $3 \mathrm{D}$ exhibits no additional features if wave propagation can be neglected [25] (i.e. the velocities are small compared to the speed of sound in the elastic bodies) and the surfaces of the contacting 
bodies obey the restrictions of the half-space approximation. Moreover, it should be pointed out that in the present work the dissipation due to plastic or viscoelastic deformations, which also can be of utmost importance in erosive wear, are disregarded to simplify the analysis.

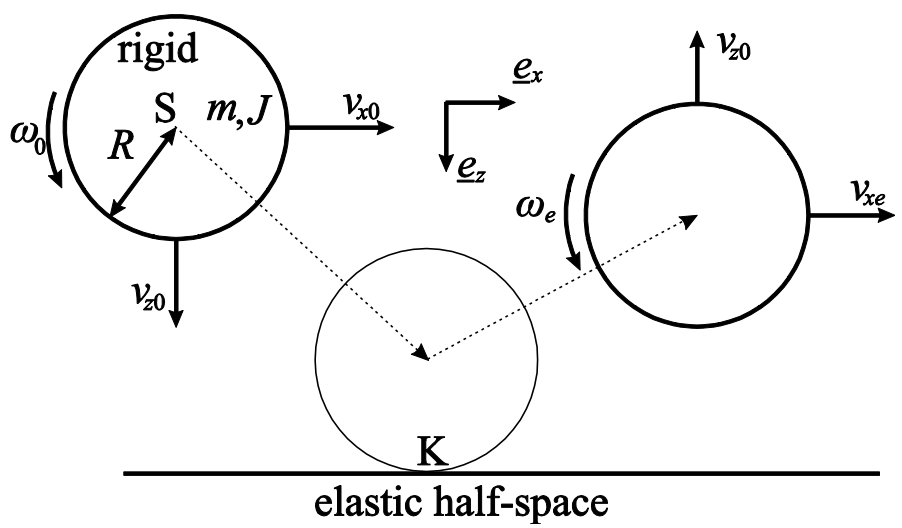

Fig. $12 \mathrm{D}$ oblique impact of a rigid sphere onto an elastic half-space. Schematic representation and notations

Under the assumptions stated above, the tangential velocity and spin after the collision can be calculated in terms of the coefficient of tangential restitution $e_{x}$,

$$
e_{x}:=-\frac{v_{x e}+s_{e}}{v_{x 0}+s_{0}}
$$

and the non-dimensional gyration parameter

$$
\kappa:=\frac{J}{J+m R^{2}}
$$

as follows:

$$
\begin{aligned}
s_{e} & =s_{0}-\left(1+e_{x}\right)(1-\kappa)\left(s_{0}+v_{x 0}\right), \\
v_{x e} & =v_{x 0}-\kappa\left(1+e_{x}\right)\left(s_{0}+v_{x 0}\right) .
\end{aligned}
$$

Hence, the loss of kinetic energy during the collision is given by

$$
\Delta E=-\frac{m}{2}\left(v_{x 0}+s_{0}\right)^{2} \kappa\left(1-e_{x}^{2}\right) .
$$

According to the energy-based approach, the total worn volume after one impact will be

$$
\Delta V=k \frac{\Delta E}{\sigma_{0}},
$$

with material hardness $\sigma_{0}$ and non-dimensional wear coefficient $k$. 
Obviously, all the macroscopic impact characteristics are known if the tangential restitution coefficient can be determined. This is done in the following section.

\section{THE COEFFICIENT OF TANGENTIAL RESTITUTION}

Maw, Barber \& Fawcett [12] have shown that, if the friction force is calculated based on the contact solutions by Mindlin \& Deresiewicz, the coefficient of tangential restitution will be a function of only two dimensionless parameters

$$
\chi:=\frac{M}{2 \kappa}, \quad \psi:=\frac{M \tan \alpha}{\mu}, \quad \text { with } \quad M:=\frac{2-2 v}{2-v} \quad \text { and } \quad \tan \alpha:=\frac{\left|v_{x 0}+s_{0}\right|}{v_{z 0}},
$$

with Poisson ratio $v$ of the elastic half-space and the coefficient of friction $\mu . M$ is the ratio of tangential to normal stiffness of the cylindrical flat punch contact, often attributed to Mindlin, and $\alpha$ the generalized impact angle accounting for pre-spin of the sphere. To spare the less important parameter, we will assume a homogeneous sphere (i.e. $\kappa=2 / 7$ ) and a constant Poisson ratio of $v=1 / 3$. Hence, $\chi=$ constant $=1.4$.

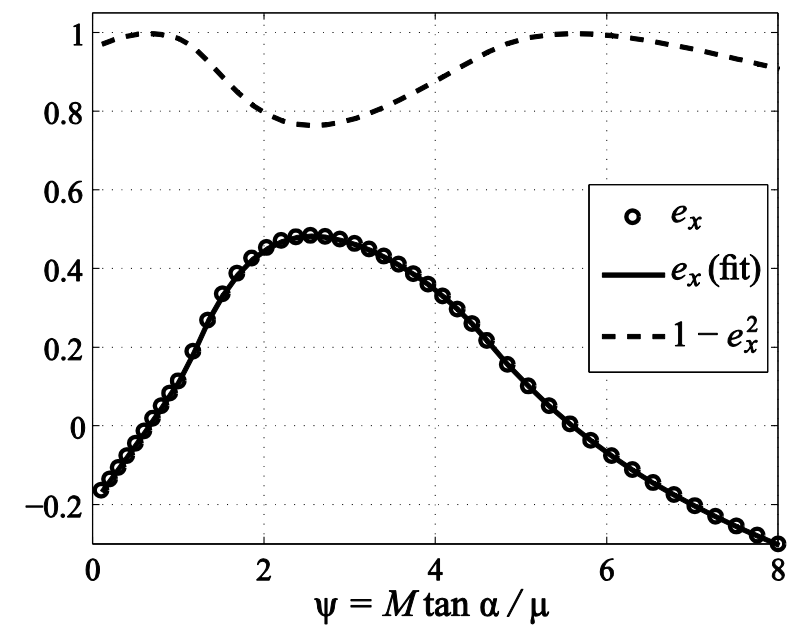

Fig. 2 Coefficient of tangential restitution $e_{x}$ as a function of $\psi$ for the oblique impact of elastic spheres with $\kappa=2 / 7$ and $v=1 / 3$, together with the analytic expressions from Eqs. (7) and (8); also shown is the relevant term for the loss of kinetic energy

The solution for $e_{x}=e_{x}(\psi)$ resulting from MBF theory needs to be calculated numerically and is shown in Fig. 2. There are three different regimes: For $\psi<1$ the impact starts with a configuration of local stick; for $1<\psi<4 \chi-1$ the impact starts with a phase of gross slip, which ends during the collision; for $\psi>4 \chi-1$ the contact is fully sliding during the whole impact and the coefficient of tangential restitution is therefore elementarily given by 


$$
e_{x}^{\mathrm{fs}}=\frac{4 \chi}{\psi}-1, \quad \text { if } \quad \psi>4 \chi-1 .
$$

In the other two regimes, for $\chi=1.4$ the solution can be approximated very well by the analytic expressions

$$
\begin{aligned}
& e_{x} \approx 0.0193 \psi^{2}+0.2896 \psi-0.1937 \quad \text { if } \quad \psi<1, \\
& e_{x} \approx-0.0081 \psi^{4}+0.115 \psi^{3}-0.6585 \psi^{2}+1.6541 \psi-1.0152 \text { if } 1<\psi<4.6 .
\end{aligned}
$$

\section{SELECTED RESUltS FOR THE LOST PORTION OF KinETIC ENERGY}

In many applications the most relevant quantity will not be the absolute value of dissipated energy but rather the portion of initial kinetic energy, which is lost during one impact. Normalizing Eq. (4) for initial kinetic energy $E_{0}$ we obtain

$$
\frac{|\Delta E|}{E_{0}}=\frac{\left(v_{x 0}+s_{0}\right)^{2} \kappa\left(1-e_{x}^{2}\right)}{v_{x 0}^{2}+v_{z 0}^{2}+\frac{\kappa}{1-\kappa} s_{0}^{2}} .
$$

To characterize the mode of the contact point's tangential motion and thus the effect of pre-spin one can introduce the ratio

$$
\xi:=\frac{v_{x 0}}{v_{x 0}+s_{0}} .
$$

Pre-spin plays an important role for the dissipated energy - which is self-evident for everybody who has seen a tennis ball bouncing off a court with what in sports is called "back spin" (i.e. $s_{0}>0$; as the tangential velocity of the contact point due to the rotation in this case is positive, the notion "forward spin" seems more appropriate) - because it enters both Eqs. (4) and (6). $\xi=1$ corresponds to no pre-spin at all whereas for $\xi=0$ the tangential motion of the contact point results from pure rotation. Values $\xi>1$ correspond to weak backward spin (contact point $K$ is still moving in positive $x$-direction) and values $\xi<0$ to strong backward spin (the direction of motion of $K$ changes).

Eq. (9) can be rewritten in the form

$$
\frac{|\Delta E|}{E_{0}}=\frac{\kappa\left(1-e_{x}^{2}\right)}{\xi^{2}+\cot ^{2} \alpha+\frac{\kappa}{1-\kappa}(1-\xi)^{2}} .
$$

With all other parameters fixed (especially generalized impact angle $\alpha$ ), this expression takes its maximum value at

$$
\xi_{c}=\kappa .
$$

which corresponds to weak forward spin (note, that $0<\kappa<0.5$ ).

In Fig. 3 the portion of lost kinetic energy is shown for $v=1 / 3, \kappa=2 / 7$ and $\xi=\kappa$ as a function of the two remaining parameters, the coefficient of friction and the generalized 
impact angle. As one would expect, the portion is increasing if both the impact angle and the coefficient of friction increase. However, for any fixed value of the coefficient of friction the portion of lost energy has a maximum at some certain impact angle and vice versa.

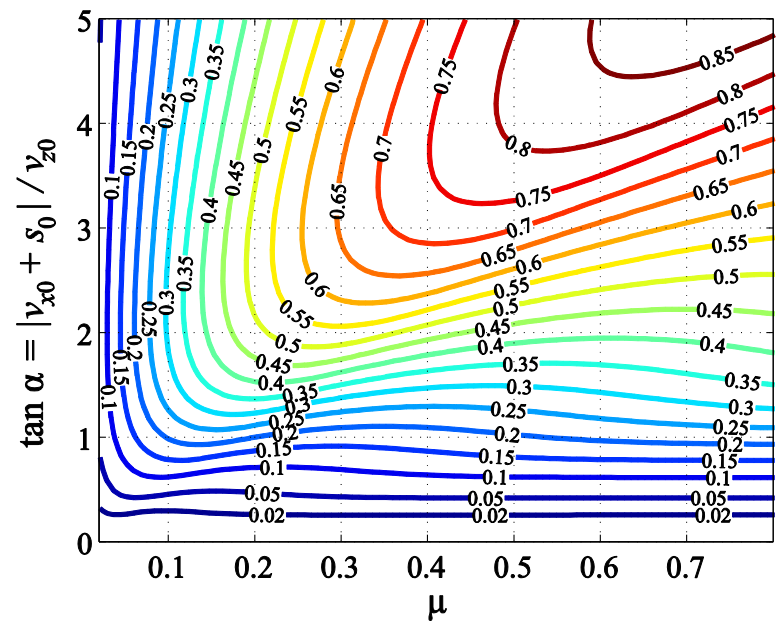

Fig. 3 Contour isoline diagram of the loss of kinetic energy normalized for the initial kinetic energy before the impact as a function of the coefficient of friction and the generalized impact angle for $v=1 / 3$ and $\kappa=2 / 7$ at the extremal configuration of tangential motion $\xi=\kappa$

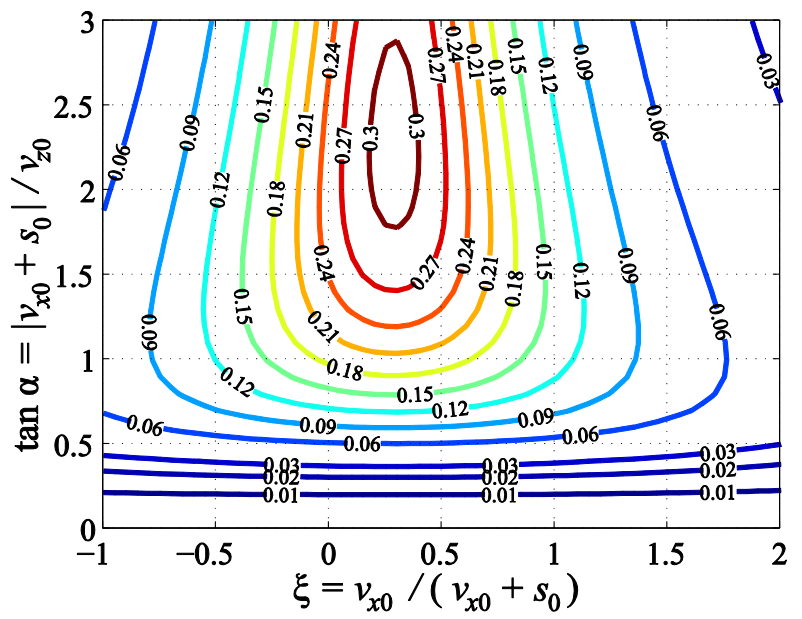

Fig. 4 Contour isoline diagram of the loss of kinetic energy normalized for the initial kinetic energy before the impact as a function of the configuration of tangential motion and the generalized impact angle for $\nu=1 / 3, \kappa=2 / 7$ and $\mu=0.1$ 
Fig. 4 and 5 visualize the normalized dissipated energy as a function of $\xi$ and the generalized impact angle for $v=1 / 3, \kappa=2 / 7$ and two values of the friction coefficient. One can clearly see how the higher losses are localized in the regions of weak forward pre-spin, around the critical value given by Eq. (12).

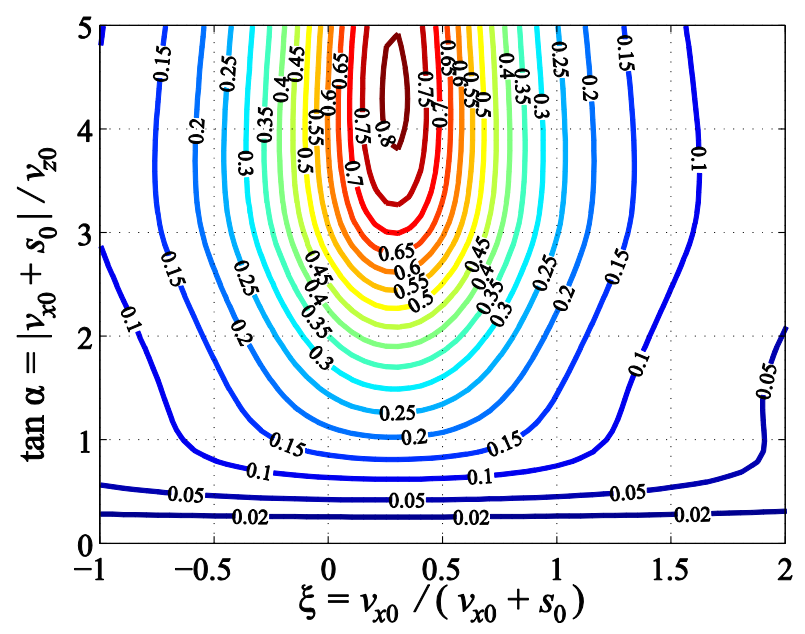

Fig. 5 Results as in Fig. 4 for $\mu=0.5$

\section{DiSTRIBUTION OF FRICTIONAL DISSIPATION OVER THE CONTACT AREA}

Not only can the total lost energy be of interest, but also its distribution over the contact area. If the energy-based wear law is valid in local form, then the distribution of frictional dissipation will give the distribution of wear and therefore the form of the impact pit after the collision (note again that plastic deformation, which will also result in a residual impact pit, is disregarded here).

During the impact the contact area (with radius $a$ ) will in general consist of an inner stick area (radius $c$ ) surrounded by an annulus of local slip. Frictional energy dissipation requires relative motion between the contacting surfaces. Hence, energy is dissipated only in the slip area. The area density of power of the frictional dissipation is given by

$$
\dot{w}(r, t)=\mu p(r, t)\left|v_{\text {rel }}(r, t)\right|, \quad c(t)<r \leq a(t),
$$

with the Hertzian pressure distribution

$$
p(r, t)=\frac{2 E^{*}}{\pi R} \sqrt{a^{2}(t)-r^{2}}, \quad r \leq a(t) .
$$

$E^{*}=E /\left(1-v^{2}\right)$, with Young's modulus $E$, is the effective Young's modulus of the elastic half-space. The relative velocity between two slipping points on the surfaces of the contacting bodies, $v_{\text {rel }}$, can be calculated from the solution of the impact problem within 
the framework of the method of dimensionality reduction (MDR; see [17] for a detailed description of the impact solution algorithm) according to

$$
\nu_{\text {rel }}(r, t)=\frac{2}{\pi} \int_{c(t)}^{r} \frac{v_{\text {rel }}^{1 \mathrm{D}}(x, t)}{\sqrt{r^{2}-x^{2}}} \mathrm{~d} x
$$

The superscript "1D" denotes the respective quantity in the MDR model. When evaluating the Abel transform (15) numerically, it is useful to follow an idea by Benad [26] and rewrite the transform via integrating by parts,

$$
v_{\text {rel }}(r, t)=v_{\text {rel }}^{1 \mathrm{D}}(r, t)-\frac{2}{\pi} \int_{c(t)}^{r} \arcsin \left(\frac{x}{r}\right) \frac{\mathrm{d}}{\mathrm{d} x}\left(v_{\text {rel }}^{1 \mathrm{D}}(x, t)\right) \mathrm{d} x,
$$

to avoid the singularity in the integrand at $x=r$.

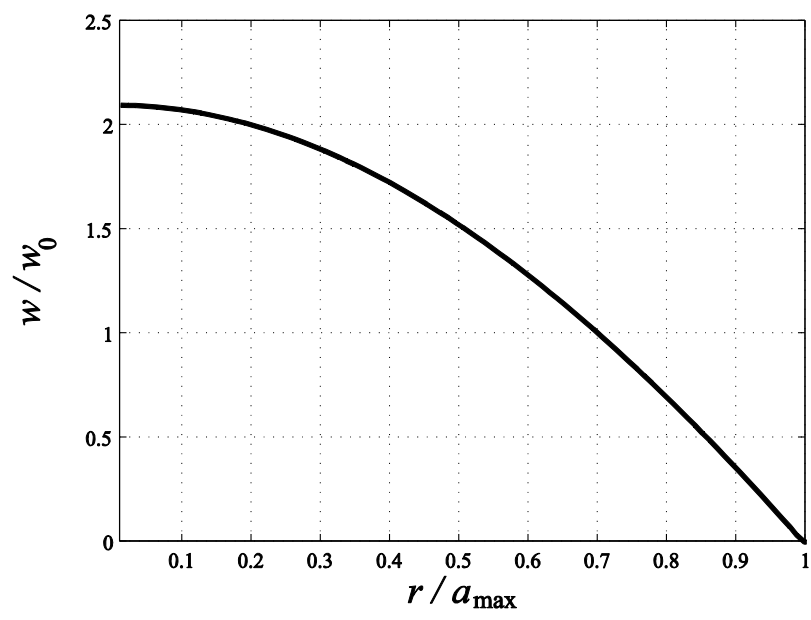

Fig. 6 Area density of frictional energy dissipation normalized for the average value as a function of the radial coordinate for full-slip collisions

Fig. 6 shows area density $w$ of the total frictional energy dissipation during the collision normalized for the average value

$$
w_{0}=\frac{|\Delta E|}{\pi a_{\max }^{2}},
$$

with the maximum contact radius during the impact, $a_{\max }$, as a function of the normalized radial coordinate $r / a_{\max }$, if the contact is fully sliding during the whole impact. In these dimensionless variables the distribution of frictional loss of energy is completely independent of the impact parameters (provided the collision indeed takes place in the full-slip regime) and can be approximated well by the expression 


$$
\frac{w^{\mathrm{fs}}(r)}{w_{0}} \approx 2.1208-0.2488 \frac{r}{a_{\max }}-1.9093\left(\frac{r}{a_{\max }}\right)^{2} .
$$

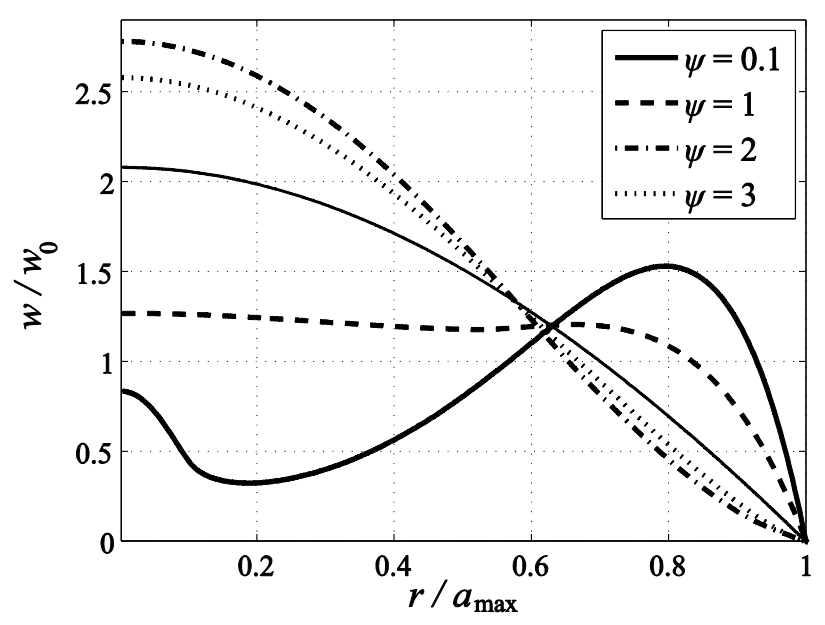

Fig. 7 Area density of frictional energy dissipation normalized for the average value as a function of the radial coordinate for several values of parameter $\psi$ and $\chi=1.4$. The thin black line denotes the full-slip case

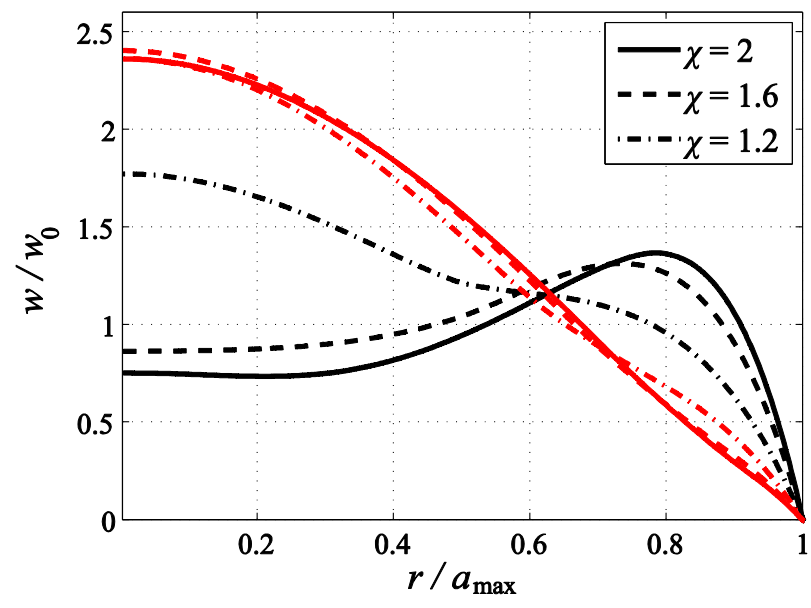

Fig. 8 Area density of frictional energy dissipation normalized for the average value as a function of the radial coordinate for several values of the parameter $\chi$. Red lines correspond to $\psi=(4 \chi-1) / 3$. Black lines correspond to $\psi=(4 \chi-1) / 5$

Generally, the normalized distribution of frictional distribution will depend on the impact parameters $\chi$ and $\psi$. In Fig. 7 the distribution is shown for the case $\chi=1.4$ (which, as said 
before, corresponds to a homogeneous sphere and $v=1 / 3$ ) and several values of $\psi$. For small values of $\psi$ (i.e. dominance of stick) the dissipation is localized away from the impact center whereas for large values (i.e. dominance of slip) the localization tends more to the center.

The closer the impact configuration is to the full-slip case, the more the dissipation density is only depending on the parameter $\psi /(4 \chi-1)$. This is demonstrated in Fig. 8. The red lines corresponding to $\psi=(4 \chi-1) / 3$ and different values of $\chi$ are very close together. On the other hand, the black lines, denoting configurations with $\psi=(4 \chi-1) / 5$ and various values of $\chi$, differ significantly from each other, especially because for $\chi=1.2$ the respective value of $\psi$ is smaller than one, i.e. the impact does not start with a phase of complete sliding.

\section{DISCUSSION}

The wear loss in impact wear has been reported in the literature to be proportional to the amount of energy dissipated during the impact [1]. Thus, in the present study the contact-impact solution for the spherical oblique elastic impact has been applied to calculate the amount of kinetic energy lost during one collision to provide an easy-to-evaluate impact wear measure. According to the analysis the main quantities that determine the impact wear volume are the coefficient of friction, the impact angle and the mode of tangential motion of the contact point (due to rotation or translation of the spheres). Thereby the portion of kinetic energy, which is lost during the collision due to frictional dissipation, has a welllocalized maximum for weak forward pre-spin.

The distribution of frictional dissipation over the contact area shows a complicated dependence on the impact parameters. For pronounced local slip (e.g. due to a small coefficient of friction) the dissipation is localized in the center of impact whereas for dominance of sticking, most energy is lost away from the center.

It should be noted that the coefficient of tangential restitution and hence the dissipated energy can be significantly influenced by elastic parameter $\chi$. The case $\chi=0.5$ is especially interesting because it can be completely energy-conserving (i.e. practically wear-less), provided that $\psi<1$. Whereas $\chi=0.5$ is impossible to realize with elastically homogeneous media due to thermodynamic stability restrictions for Poisson's ratio, dissipation-less configurations are within reach for materials with a functional elastic grading [27].

Last but definitely not least, plastic deformations during the impact will gain importance for intermediate or high impact velocities. This aspect has been neglected here. Although some approaches to tackle the elasto-plastic oblique impact problem have been published in the past years [28, 29], contact mechanically rigorous models for this problem are still scarce and thus require further investigation.

\section{REFERENCES}

1. Souilliart, T., Rigaud, E., Le Bot, A., Phalippou, C., 2017, Energy-based wear law for oblique impacts in dry environment, Tribology International, 105, pp. 241-249.

2. Tarbe, R., Kulu, P., 2008, Impact wear tester for the study of abrasive erosion and milling processes, $6^{\text {th }}$ International DAAAM Baltic Conference Industrial Engineering, Tallin

3. Finnie, I., 1960, Erosion of surfaces by solid particles, Wear, 3(2), pp. 87-103.

4. Neilson, J.H., Gilchrist, A., 1968, Erosion by a stream of solid particles, Wear, 11(2), pp. 111-122. 
5. Engel, P.A., 1978, Percussive impact wear: A study of repetitively impacting solid components in engineering, Tribology International, 11(3), pp. 169-176.

6. Talia, M., Lankarani, H., Talia, J.E., 1999, New experimental technique for the study and analysis of solid particle erosion mechanisms, Wear, 225-229 (Part 2), pp. 1070-1077.

7. Hertz, H., 1882, Über die Berührung fester elastischer Körper, Journal für die reine und angewandte Mathematik, 92, pp. 156-171.

8. Johnson, K.L., Pollock, H.M., 1994, The role of adhesion in the impact of elastic spheres, Journal of Adhesion Science and Technology, 8(11), pp. 1323-1332.

9. Thornton, C., Ning, Z., 1998, A theoretical model for the stick/bounce behaviour of adhesive, elasticplastic spheres, Powder Technology, 99(2), pp. 154-162.

10. Hunter, S.C., 1957, Energy absorbed by elastic waves during impact, Journal of the Mechanics and Physics of Solids, 5(3), pp. 162-171.

11. Ghanbarzadeh, A., Hassanour, A., Neville, A., 2018, A numerical model for calculation of the restitution coefficient of elastic-perfectly plastic and adhesive bodies with rough surfaces, Powder Technology, accepted manuscript, doi: https://doi.org/10.1016/j.powtec.2018.12.079

12. Maw, N., Barber, J.R., Fawcett, J.N., 1976, The oblique impact of elastic spheres, Wear, 38(1), pp. 101-114.

13. Mindlin, R.D., Deresiewicz, H., 1953, Elastic Spheres in Contact under Varying Oblique Forces, Journal of Applied Mechanics, 20, pp. 327-344.

14. Gorham, D.A., Kharaz, A.H., 2000, The measurement of particle rebound characteristics, Powder Technology, 112(3), pp. 193-202.

15. Lyashenko, I.A., Willert, E., Popov, V.L., 2018, Mechanics of collisions of solids: influence of friction and adhesion. I. Review of experimental and theoretical works, PNRPU Mechanics Bulletin, 2, pp. 4461, doi: http://dx.doi.org/10.15593/perm.mech/2018.2.05 .

16. Popov, V.L., Heß, M., 2015, Method of Dimensionality Reduction in Contact Mechanics and Friction, Springer-Verlag, Berlin Heidelberg, 265 p.

17. Willert, E., Popov, V.L., 2016, Impact of an elastic sphere with an elastic half space with a constant coefficient of friction: Numerical analysis based on the method of dimensionality reduction, ZAMM Zeitschrift für Angewandte Mathematik und Mechanik, 96(9), pp. 1089-1095.

18. Doménech-Carbó, A., 2013, Analysis of oblique rebound using a redefinition of the coefficient of tangential restitution coefficient, Mechanics Research Communications, 54, pp. 35-40.

19. Pishkenari, H.N., Rad, H.K., Shad, H.J., 2017, Transformation of sliding motion to rolling during spheres collision, Granular Matter, 19:70, doi: https://doi.org/10.1007/s10035-017-0755-0 .

20. Brach, R.M., 1988, Impact dynamics with applications to solid particle erosion, International Journal of Impact Engineering, 7(1), pp. 37-53.

21. Archard, J.F., Hirst, W., 1956, The wear of metals under unlubricated conditions, Proceedings of the Royal Society of London, Series A, 236, pp. 397-410.

22. Khrushchov, M.M., Babichev, M.A., 1960, Investigation of Wear of Metals, Russian Academy of Sciences, Moscow, $252 \mathrm{p}$

23. Honda, K., Yamada, K., 1925, Some experiments on the abrasion of metals, Journal of the Institute of Metals, 33(1), pp. 49-68

24. Argatov, I.I., Dmitriev, N.N., Petrov, Y.V., Smirnov, V.I., 2009, Threshold erosion fracture in the case of oblique incidence, Journal of Friction and Wear, 30(3), pp. 176-181.

25. Hunter, S.C., 1957, Energy absorbed by elastic waves during impact, Journal of the Mechanics and Physics of Solids, 5(3), pp. 162-171.

26. Benad, J., 2018, Fast numerical implementation of the MDR transformations, Facta Universitatis, Series Mechanical Engineering, 16(2), pp. 127-138.

27. Willert, E., Popov, V.L., 2017, The oblique impact of a rigid sphere on a power-law graded elastic halfspace, Mechanics of Materials, 109, pp. 82-87.

28. Ghaednia, H., Marghitu, D.B., 2016, Permanent deformation during the oblique impact with friction, Archive of Applied Mechanics, 86(1-2), pp. 121-134.

29. Wu, Y.-C., Thornton, C., Li, L.-Y., 2009, A semi-analytical model for oblique impacts of elastoplastic spheres, Proceedings of the Royal Society of London, Series A, 465, pp. 937-960. 